\title{
Article
}

\section{Bi-Directional Origami-Inspired SMA Folding Microactuator}

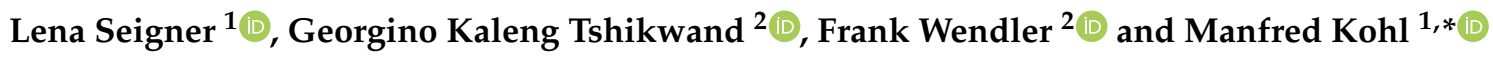 \\ 1 Institute of Microstructure Technology, Karlsruhe Institute of Technology, \\ 76344 Eggenstein-Leopoldshafen, Germany; lena.seigner@kit.edu \\ 2 Institute of Materials Simulation, Friedrich-Alexander University of Erlangen-Nürnberg (FAU), \\ 90762 Fürth, Germany; georgino.tshikwand@fau.de (G.K.T.); frank.wendler@fau.de (F.W.) \\ * Correspondence: manfred.kohl@kit.edu
}

Citation: Seigner, L.; Tshikwand,

G.K.; Wendler, F.; Kohl, M.

Bi-Directional Origami-Inspired SMA

Folding Microactuator. Actuators 2021,

10, 181. https://doi.org/10.3390/

act10080181

Academic Editor: Wei Min Huang

Received: 22 June 2021

Accepted: 27 July 2021

Published: 3 August 2021

Publisher's Note: MDPI stays neutral with regard to jurisdictional claims in published maps and institutional affiliations.

Copyright: (c) 2021 by the authors. Licensee MDPI, Basel, Switzerland. This article is an open access article distributed under the terms and conditions of the Creative Commons Attribution (CC BY) license (https:/ / creativecommons.org/licenses/by/ $4.0 /)$.

\begin{abstract}
We present the design, fabrication, and characterization of single and antagonistic SMA microactuators allowing for uni- and bi-directional self-folding of origami-inspired devices, respectively. Test devices consist of two triangular tiles that are interconnected by double-beam-shaped SMA microactuators fabricated from thin SMA foils of $20 \mu \mathrm{m}$ thickness with memory shapes set to a $180^{\circ}$ folding angle. Bi-directional self-folding is achieved by combining two counteracting SMA microactuators. We present a macromodel to describe the engineering stress-strain characteristics of the SMA foil and to perform FEM simulations on the characteristics of self-folding and the corresponding local evolution of phase transformation. Experiments on single-SMA microactuators demonstrate the uni-directional self-folding and tunability of bending angles up to $180^{\circ}$. The finite element simulations qualitatively describe the main features of the observed torque-folding angle characteristics and provide further insights into the angular dependence of the local profiles of the stress and martensite phase fraction. The first antagonistic SMA microactuators reveal bi-directional self-folding in the range of $-44^{\circ}$ to $+40^{\circ}$, which remains well below the predicted limit of $\pm 100^{\circ}$.
\end{abstract}

Keywords: self-folding origami; microactuation; shape memory alloy foils; micro technology

\section{Introduction}

The increasing demand on the functionality of microsystems requires new approaches for active control of mechanical, optical, and fluidic components. Most microactuator concepts are limited to one- or two-dimensional workspaces. The concept presented here uses the technique of self-folding, following the ancient art of paper folding, known as origami, by which flat sheets transform into numerous three-dimensional (3D) shapes. The shape change can be achieved by bimorph structures [1], smart composites [2,3], or smart materials such as shape memory alloys (SMAs), responding to an external stimulus such as the environmental temperature or an electrical current. The advantage of origamiinspired designs in engineering is their compact and deployable setup, which has proven its versatility in various macroscopic applications, e.g., airbags [4], wings [5], or tools for minimally invasive surgery [6]. In addition, the actual 3D function could be assigned after the fabrication of the initially flat 2D structures. An overview of the designs and mechanisms of self-folding structures can be found in recent reviews, e.g., [7-9].

SMAs provide high bending moments, low corrosion, and low fatigue. SMA wires have been used to produce a folding motion [10-12] and furthermore can be transformed into SMA coils to generate larger displacements and bending angles $[6,13]$. The use of SMA sheets or foils can simplify the design and fabrication of folding actuators at the miniature scale [14]. Previous concepts of origami-inspired self-folding SMA sheets have been published in $[10,15]$. Further relevant research has been performed, e.g., in the field of robogami [16]. Alternative mechanisms for satellite deployment using SMA hinges were presented in [17]. While previous developments focused on the macro scale, we intend to transfer the concept of origami-inspired SMA folding actuation to the micro scale by 
combining state-of-the-art methods of SMA film engineering and micromachining. The aim of this study is to design, fabricate, and analyze the performance of single and antagonistic SMA microactuators, allowing for uni- and bidirectional folding at miniature scales.

\section{Materials and Methods}

\subsection{Materials Characterization}

The outstanding property of SMAs is their thermally induced solid-state phase transformation, from a low-temperature phase (martensite) to a high-temperature phase (austenite) [18]. When an SMA material is deformed in its martensitic state, it undergoes a diffusionless and reversible phase transformation. Restoring its memory shape requires heating above its phase transformation temperature (austenite finish temperature $A_{f}$ ). In this investigation, the base material is a cold-rolled NiTi foil of $20 \mu \mathrm{m}$ thickness. Aiming at multi-physical simulation of the folding actuation, the material is characterized with regard to its mechanical, thermal, and electrical properties. The differential scanning calorimetry (DSC) measurement shown in Figure 1 provides information on the martensitic transformation temperatures of the as-received material. The phase transformation regime is roughly located between 5 and $62{ }^{\circ} \mathrm{C}$. On cooling, the material undergoes an intermediate $\mathrm{R}$-phase transformation before the transformation to martensite begins at approximately $19^{\circ} \mathrm{C}$. Therefore, at room temperature, the material is in R-phase condition as long as no external load is applied. The reverse transformation from $R$-phase to austenite is associated with low thermal hysteresis and excellent fatigue life $[19,20]$. This behavior is reflected in the electrical resistance characteristic in Figure $1 \mathrm{~b}$ showing the temperature range of folding actuation above room temperature. After the first load cycle, phase transformation is highly reversible.

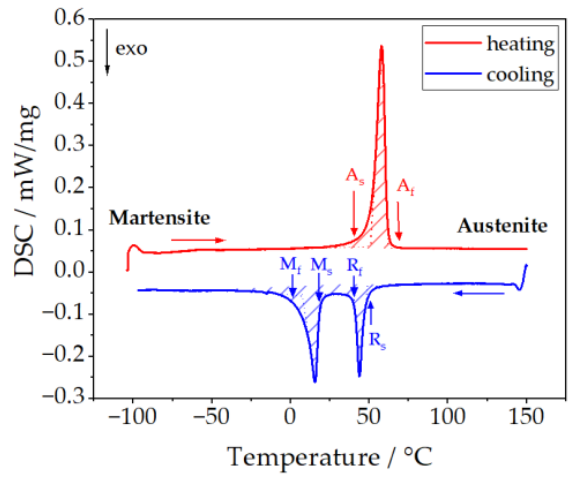

(a)

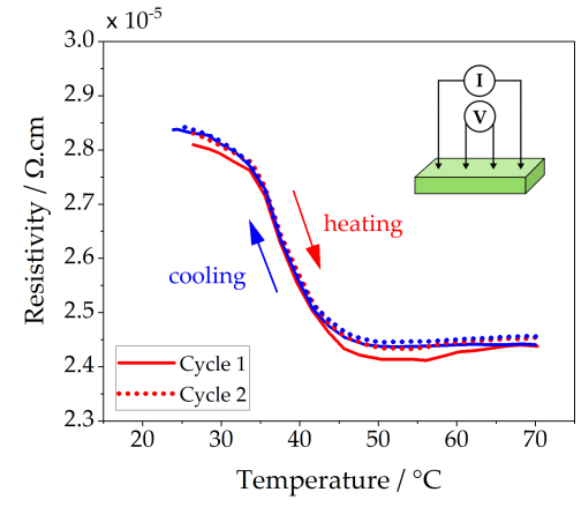

(b)

Figure 1. (a) Differential scanning calorimetry measurement of a cold-rolled NiTi foil of $20 \mu \mathrm{m}$ thickness; (b) four-point-probe electrical resistivity measurement in the temperature regime of actuation above room temperature.

Tensile tests are carried out in a heating chamber in order to determine the temperaturedependent mechanical parameters including Young's modulus, maximum transformation strain, and critical stress for inter-martensitic transformation. Figure 2a shows experimental and simulated engineering stress-strain characteristics for different temperatures. Typical features are the increase in stiffness and of the critical transformation stress, which is apparent by the shift of the stress plateau toward higher values. The stiffness increase is attributed to the rule of mixture of the involved phase fractions, including the 'soft' $\mathrm{R}$-phase and 'hard' austenite that change with temperature. The critical transformation stress increases linearly following the Clausius Clapeyron relation with coefficient $C_{\mathrm{AM}}$. The experimental characteristic at room temperature exhibits a rather large slope of the stress plateau due to strain hardening caused by sample fabrication. The obtained material parameters are summarized in Table A1 in the Appendix A. 


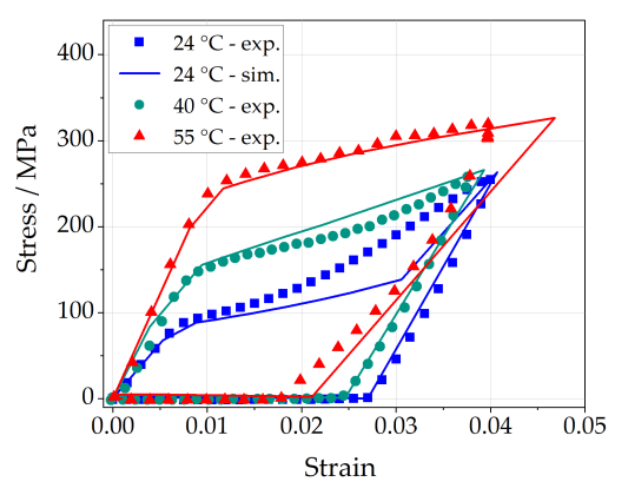

(a)

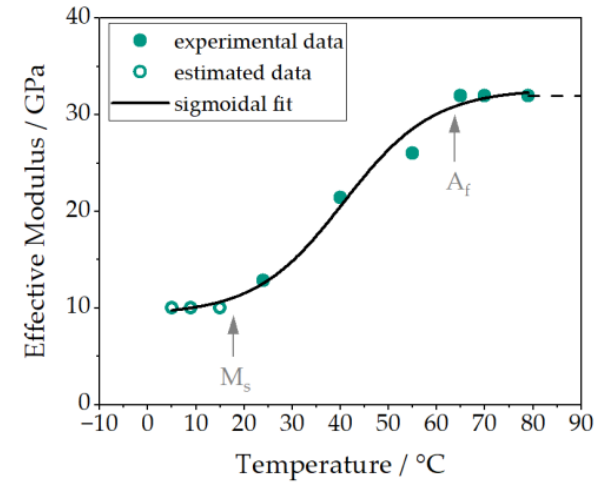

(b)

Figure 2. (a) Experimental and simulated stress-strain characteristics at different temperatures as indicated; (b) temperature-dependent effective modulus used in the present model to describe the mechanical response in the temperature regime of co-existing $\mathrm{R}$ - and A-phase.

\subsection{Constitutive Modeling}

The model used to describe the thermomechanical behavior of the SMA material has been adopted from Jaber et al. [21]. Contrary to other polycrystalline model approaches, it is based upon the strain tensor and temperature as control variables for the transformation process. In combination with the finite element method for finite strains and large displacements, this model has a very good convergence behavior. The change from a $(\sigma, T)$ - to an equivalent $(\varepsilon, T)$-phase diagram is necessary to determine the transformation rates directly expressed by the strain rate. Skipping the stress calculation in the adopted algorithm reduces computation time. Similar to other phenomenological material models for SMAs, the present model consists of two laws: a thermomechanical and a kinetic law. Here, we present the general equations and refer the reader to references [21-23] for further details.

During the thermomechanical formulation, hypo-elastic plasticity modeling is conducted to describe the non-linear but reversible stress-strain behavior [24]. Here, the strain rate tensor is additively decomposed, as in the equation below,

$$
\dot{\varepsilon}=\dot{\varepsilon}_{e l}+\dot{\varepsilon}_{t r}
$$

$\dot{\varepsilon}$ is the rate of the total strain, $\dot{\varepsilon}_{e l}$ is the rate of the elastic strain, and $\dot{\varepsilon}_{t r}$ is the rate of the transformation strain. Considering the Jaumann rate of the Cauchy stress as the stress measure, the thermomechanical law is stated as below,

$$
\dot{\boldsymbol{\sigma}}=\boldsymbol{R}: \dot{\varepsilon}_{e l}+\boldsymbol{k} \dot{T}=\boldsymbol{R}:\left(\dot{\varepsilon}-\dot{\varepsilon}_{t r}\right)+\boldsymbol{k} \dot{\boldsymbol{T}}
$$

$T$ is the current temperature, $k$ is the thermal modulus tensor, and $R$ is the fourth-order elastic modulus.

The martensite volume fraction $\xi$ and transformation strain tensor $\varepsilon_{t r}$ are selected as the internal state variables. These averaged quantities give a global account of the material behavior during thermomechanical loading. The kinetic law, therefore, describes the evolution of the internal state variables as below,

$$
\dot{\varepsilon}_{t r}=\dot{\xi} \boldsymbol{E},
$$

where $E$ is the transformation direction tensor that controls the orientation of created or recovered transformation strain for both forward and reverse transformations. $E$ is defined as,

$$
E=\varepsilon_{t r}^{\max } \frac{\sigma^{\prime}}{\left\|\sigma^{\prime}\right\|} \text { for } \dot{\xi}>0 \text { (forward transformation) }
$$




$$
E=\varepsilon_{t r}^{\max } \frac{\varepsilon_{t r}}{\left\|\varepsilon_{t r}\right\|} \text { for } \dot{\xi}<0 \text { (reverse transformation) }
$$

here, $\|\varepsilon \prime\|=\sqrt{\frac{3}{2(1+v)^{2}} \varepsilon^{\prime}: \varepsilon \prime}$ indicates the tensor norm.

In forward transformation, the created strain follows the direction of the deviator $\sigma^{\prime}$, which controls shape change in the materials. In reverse transformation, the strain decrease follows the direction of the existing transformation strain $\varepsilon_{t r}$. With this constitutive relation, a tangent stiffness tensor is calculated, which relates local stress and strain increments. In the finite element implementation, an elastic predictor-transformation corrector return mapping algorithm is adopted. Geometric nonlinearity is treated with an updated Lagrangian formulation [25].

The phase diagram in Figure 3 has been obtained from evaluating the tensile loading data (see Table A1). In the model, we take a simplified view: In forward transformation, we assume the co-existence of R- and A-phase, which we combine to a single parent phase and describe by a temperature-dependent effective modulus determined from experimental material characterization. Above $A_{f}$, the stiffness of the unloaded material is constant, and we estimate the modulus of the starting phase to be $10 \mathrm{GPa}$ below room temperature. Figure $2 \mathrm{~b}$ depicts both the experimental and fitted effective modulus for different temperatures. The present model assumes the same mechanical behavior for tension and compression. In various NiTi alloys, a pronounced tension-compression asymmetry was found with typically higher transformation stress levels and lower transformation strain under compressive load [26]. An adaption of the model parameters will be possible in the future when experimental bending tests have been carried out.

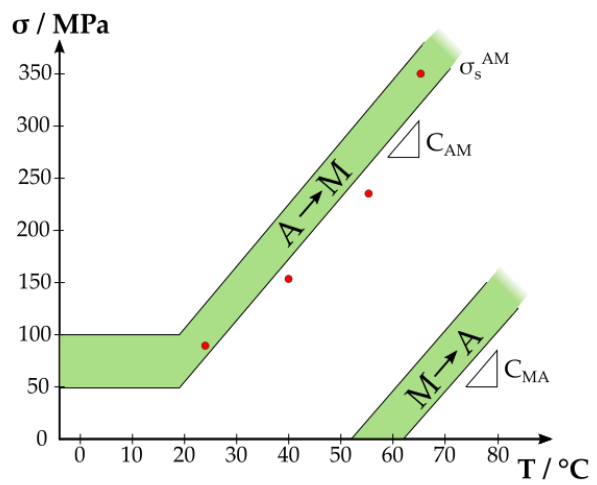

(a)

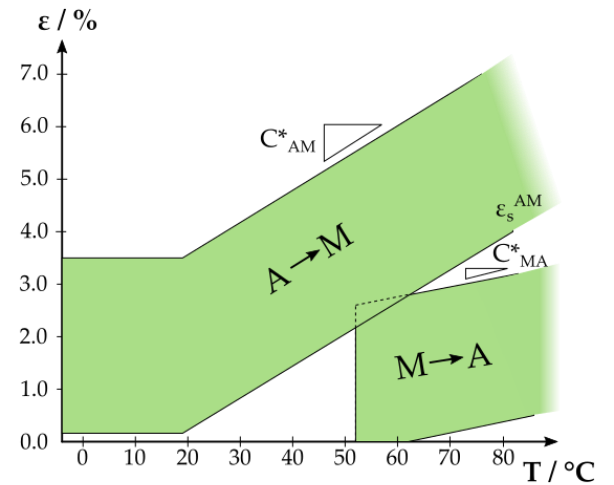

(b)

Figure 3. (a) Classical phase diagram obtained from experimental values (red dots); (b) conversion into modified phase diagram as adapted from [21].

\subsection{Design and Engineering}

The basic components of the origami-inspired microdevices are two triangular tiles that are interconnected by SMA folding microactuators. Each microactuator is designed as a double-beam structure as depicted in Figure $4 a$, which allows for resetting the memory shape by Joule heating and thereby localizing the heat to the folding region. While eliminating the need for an additional heating element, the double-beam structure also serves as a flexible active hinge connecting the individual tiles. The dimensions of the folding microactuator are mainly limited by the SMA foil thickness $t_{S M A}$. If $\varepsilon_{\max }$ denotes the maximum allowable tensile and compressive strain, which is located on the outer surfaces of the folding area, we can calculate the minimum admissible bending radius $r$ as follows:

$$
r>t_{S M A} \frac{1-\left|\varepsilon_{\max }\right|}{2\left|\varepsilon_{\max }\right|}
$$




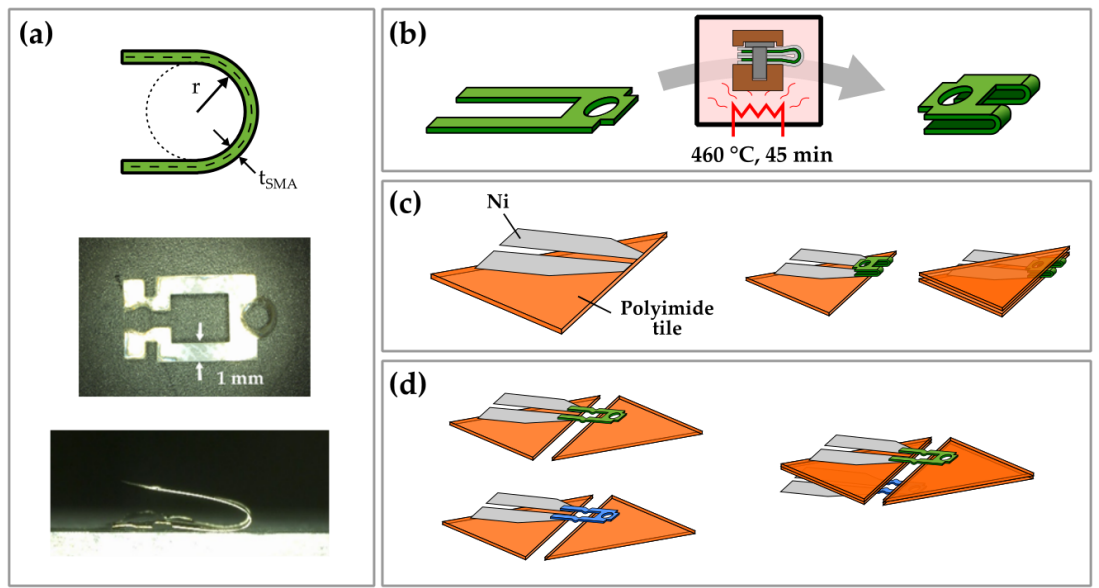

Figure 4. Fabrication and assembly of the folding microactuators: (a) microscope images of the micromachined NiTi foil before and after shape setting. (b) Schematic of laser micromachining and shape setting in a vacuum oven and (c) of electrical connection by gap welding and bonding to tiles. (d) Schematic of combining a protagonist and an antagonist microactuator for bi-directional folding.

For the given NiTi foil thickness of $20 \mu \mathrm{m}$ and a strain limit of around $6 \%$, the minimum bending radius is $157 \mu \mathrm{m}$. For our samples, we chose a bending radius of $250 \mu \mathrm{m}$ to stay safely below the strain limit. We thereby obtained a minimum separation of the tiles of $\pi\left(\boldsymbol{r}+\boldsymbol{t}_{S M A}\right)=0.82 \mathrm{~mm}$. The beam width was set to $1 \mathrm{~mm}$ and the beam separation to $2.5 \mathrm{~mm}$. Additionally, two centering holes were designed, which are required later in the heat treatment procedure.

\subsection{Fabrication}

The manufacturing process of the folding microactuators is shown in Figure $4 \mathrm{~b}-\mathrm{d}$ ). First, the NiTi foil is structured by laser cutting. The obtained structure is covered with titanium foils to prevent oxidation during the annealing step. By means of a pin and the centering holes, we can bring the stack of Ti and NiTi foils into the desired fold of $0^{\circ}$ and a folding diameter of $500 \mu \mathrm{m}$. Then, shape setting is performed by heat treatment in a vacuum furnace at $460^{\circ} \mathrm{C}$ for $45 \mathrm{~min}$. In the next step (Figure $4 \mathrm{c}$ ), the NiTi microactuators have to be attached to the polyimide tiles, which is performed here by adhesive bonding. Two nickel sheets are used as electrical connections, which are welded to the legs of the NiTi structure. The second tile is finally bonded to the NiTi structure to obtain the base element of the folding microactuator. Combining a protagonist microactuator with memory shape at $0^{\circ}$ with an antagonist with memory shape at $360^{\circ}$ (Figure $4 \mathrm{~d}$ ) enables bi-directional folding.

\section{Characterization of Folding Actuation}

The SMA folding actuator deforms quasi-plastically upon loading due to intermartensitic transformation. As shown in Figure 5, the shape-set folding microactuator has been mechanically unfolded beyond $360^{\circ}$ at room temperature to achieve a visible deformation of approximately $210^{\circ}$. If the structure is then placed on a hot plate, the memory shape can be recovered by heating above $\mathrm{A}_{\mathrm{f}}$ temperature. On subsequent cooling, the structure opens up again to about $45^{\circ}$ due to internal stress induced by the large bending angle. Repeatable performance is achieved after five training cycles. However, no systematic investigation has been made into the fatigue life.

\subsection{Uni-Directional Self-Folding}

In the following, we present the results of the folding performance of a single SMA microactuator, which is quasi-plastically deformed to $180^{\circ}$ and subsequently restored to its memory shape by Joule heating. The motion and temperature of the microactuator are tracked by an infrared camera. Different load cases are considered, ranging from free 
recovery, whereby no tiles are used, to the cases of Kapton and ceramic tiles, generating different gravitational forces. Figure 6 depicts the corresponding folding characteristics as a function of heating power, which correlates with the average temperature of the microactuator. For increasing load, the critical power to start the transformation shifts from about 150 to $500 \mathrm{~mW}$. In all cases, full transformation is reached at a heating power of $600 \mathrm{~mW}$. The corresponding critical temperatures increase from about 32 to $48{ }^{\circ} \mathrm{C}$ for the ceramic tile, which is due to the stress-induced increase of phase transformation temperatures. At the same time, the folding angle drops more steeply. Upon cooling, the memory shape is recovered completely due to the gravitational load of the ceramic tile.
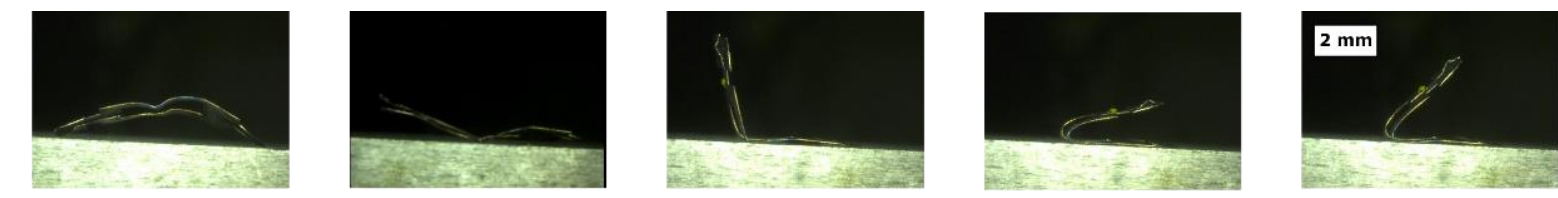

\section{$23{ }^{\circ} \mathrm{C}$}

\section{$80^{\circ} \mathrm{C}$}

Figure 5. Folding sequence of a mechanically deformed SMA folding microactuator at room temperature. The memory shape can be recovered by heating on a hot plate above the austenite finish temperature.

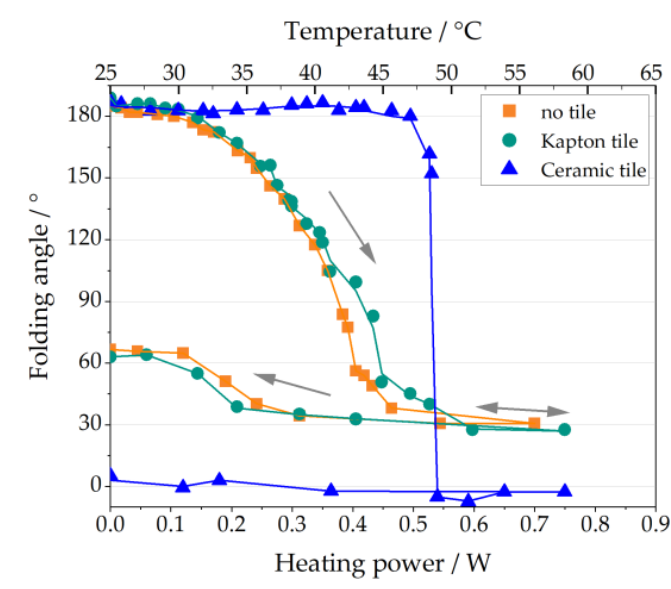

(a)

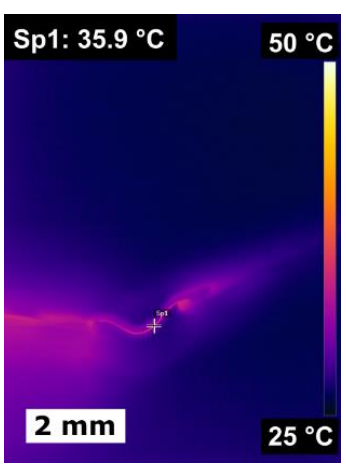

(b)

Figure 6. (a) Folding angles as a function of heating power and average temperature of the selffolding microactuator determined by camera tracking; (b) IR image taken during shape recovery.

These measurements prove the concept of folding and the tunability of the angular range by introducing a suitable additional load. However, at a small load, incomplete shape recovery is observed as a consequence of residual plastic deformation. Additional experiments prove that samples with an increased folding radius of 0.5 and $1 \mathrm{~mm}$ show less residual strain and, thus, a smaller difference between the folding angles in a hot and cold state. On the other hand, shape setting results in smaller folding angles in this case, as higher bending radii are associated with smaller strain during mechanical unfolding, causing reduced shape recovery [27].

In addition, we investigate the torque of the SMA microactuator through the blocking force that is generated during shape recovery. For this purpose, the SMA microactuator is positioned in two configurations $\left(90^{\circ}\right.$ and $\left.180^{\circ}\right)$ below a load cell with one end fixed onto a substrate (see Figure 7a). The shape recovery during heating is blocked by the load cell, and the acting force is measured. The torque is calculated by multiplying the measured blocking force of the load cell with the distance $\mathrm{L}$ with respect to the fixation. Figure $7 \mathrm{~b}$ shows the heating and cooling characteristics for each configuration. The observed hysteresis is attributed to the difference between the forward and reverse phase transformation 
temperatures. The maximum generated torque is determined to be 0.085 and $0.11 \mathrm{Nmm}$ for the folding angles of $90^{\circ}$ and $180^{\circ}$, respectively.

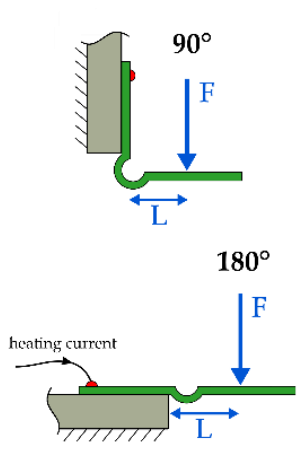

(a)

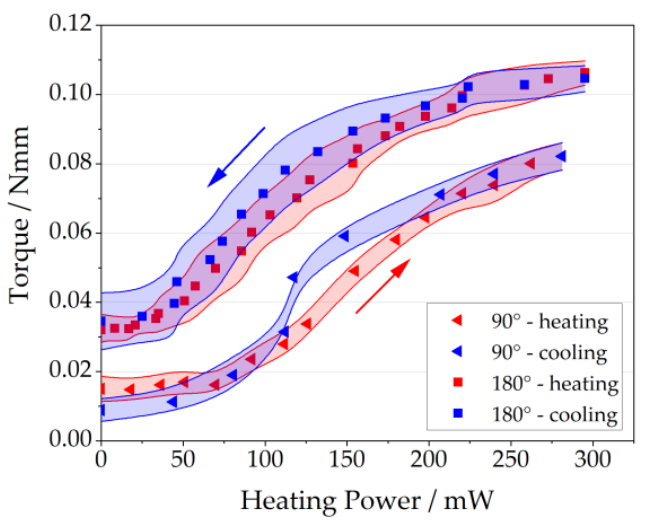

(b)

Figure 7. Measurement of blocking force versus folding angle of a single self-folding microactuator: (a) Schematic of experimental setup for the case of $90^{\circ}$ and $180^{\circ}$ folding angle; the resulting torque is obtained by the product of measured force $\mathrm{F}$ and distance $\mathrm{L}$ with respect to the fixation; (b) torque versus heating power characteristics upon heating and cooling.

\subsection{Bi-Directional Self-Folding}

By combining two counteracting microactuators, a protagonist and an antagonist, bi-directional self-folding can be achieved. Thereby, selective heating of only one microactuator at a given time is mandatory to avoid plastic deformation in the austenitic state. While the active actuator is heated, the antagonist in a cold state will be deformed. First, we determine the torque-folding angle characteristics of a single SMA microactuator at room temperature and under Joule heating. As in the previous experiment on single microactuators, the load cell is positioned above the actuator (Figure 8a), and the fixation of the tile is gradually rotated to adjust different folding angles. Using digital image processing, we evaluate the folding angle and the distance from the pin to fixation in order to derive the corresponding torque to the recorded blocking force. Figure $8 \mathrm{~b}$ shows torque-folding angle characteristics for different average temperatures of the microactuators. The experimental setup allows for limited accuracy at small folding angles. In the initial elastic range, the stiffness increases for increasing average temperature. Joule heating up to $60{ }^{\circ} \mathrm{C}$ using a heating power of $110 \mathrm{~mW}$ causes only partial phase transformation. In this case, stress-induced formation of martensite causes a characteristic change in slope above a critical torque of about $0.55 \mathrm{Nmm}$ and a plateau-like course of torque. Further heating to $80^{\circ} \mathrm{C}$ results in full transformation to austenite, which is reflected by the quasi-linear loading characteristic. Yet, the folding microactuator is deformed plastically upon loading. Therefore, residual strain occurs upon unloading, which cannot be recovered.

The FE model is applied to the bending of a single self-folding microactuator to understand the folding motion and examine the torque in the range of small folding angles. For the 3D structure (Figure 9a), a zero-displacement boundary condition is applied to fix the two legs (green) to the tile below. For the blue part connecting the two beams to the second tile, a rigid body constraint is used, and unfolding is simulated by linearly increasing the moment on this part. Figure $9 \mathrm{~b}$ depicts two different configurations $\left(180^{\circ}\right.$ and $\left.270^{\circ}\right)$ and the corresponding volume distribution of martensite phase fraction at a homogeneous temperature of $24^{\circ} \mathrm{C}$. We can observe that the phase transformation does not occur uniformly along the folding axis but starts on the outer surfaces of the microactuator. For a folding angle of $270^{\circ}$, the maximum von Mises stress is $217 \mathrm{MPa}$ and the maximum principal strain is $4.3 \%$. 


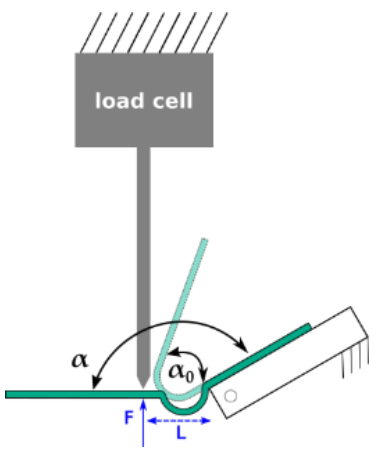

(a)

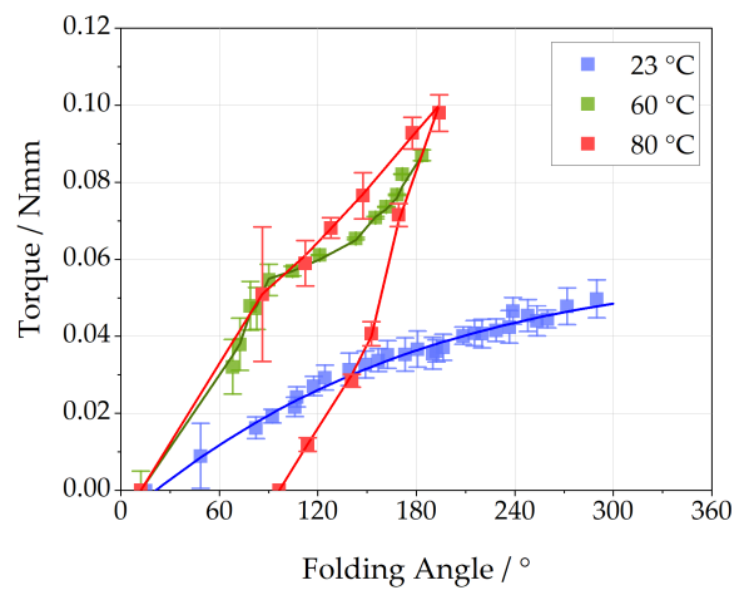

(b)

Figure 8. Measurement of blocking force versus folding angle of a single self-folding microactuator at different temperatures: (a) schematic of experimental setup; (b) torque-folding angle characteristics for different average temperatures of the microactuator.

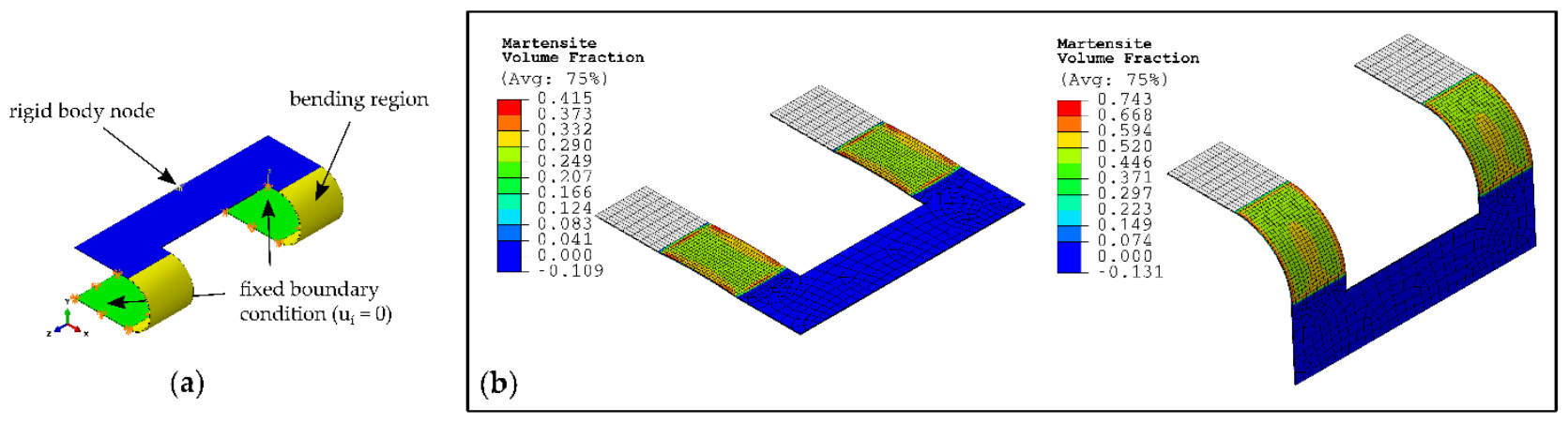

Figure 9. Finite element simulation of moment-controlled unfolding of a single microactuator at $24{ }^{\circ} \mathrm{C}$ : (a) boundary conditions of the 3D structure; (b) two folding configurations and corresponding local profile of martensite phase fraction indicated in color.

Simulations of torque-folding angle characteristics are carried out for different homogeneous temperatures, as shown in Figure 10a. Close to $160^{\circ}$, a kink occurs, which indicates a buckling effect that becomes increasingly pronounced for increasing temperature when the structure becomes stiffer. Figure 10b depicts the corresponding changes in martensite volume fraction in the bending region, covering the section between the fixation of the tiles and the connection of the two beams, see Figure 9 . At $80^{\circ} \mathrm{C}$, the microactuator remains in an austenitic state, even for folding angles up to $180^{\circ}$. For larger deformation loads, stress-induced formation of oriented martensite occurs, which is fully reset upon unloading. At lower temperatures, stress-induced transformation to oriented martensite is initiated at lower folding angles between $90^{\circ}$ and $180^{\circ}$. Thus, higher fractions of martensite occur for a given folding angle in this range. Below about $60^{\circ} \mathrm{C}$, oriented martensite can no longer be fully reset and, thus, folding angles are no longer recovered upon unloading. An animation of unfolding as well as the corresponding evolution of martensite phase fraction and von Mises stress for the cases of 24 and $80{ }^{\circ} \mathrm{C}$ can be found in videos S1-S4 of the Supplementary Materials, respectively.

Based on the torque-folding angle characteristics, the motion range of antagonistic self-folding microactuators can be predicted. Figure 11 shows torque versus folding angle of bi-directional microactuation for the case of $180^{\circ}$ pre-deflection of protagonist and antagonist, thus a total of $360^{\circ}$ with respect to each other. Zero position corresponds to a flat state (Position 1). The intersections of the characteristics of protagonist and 
antagonist correspond to the equilibrium positions obtained when selectively heating one microactuator at a given time. When the protagonist is heated exclusively, the folding angle $\alpha$ changes to Position 2 at $-100^{\circ}$, while selective heating of the antagonist results in a folding angle at $+100^{\circ}$ (Position 3). Thus, the equilibrium positions mark the maximum possible range of folding angles that could be achieved by antagonistic actuation. In an ideal case, bi-directional actuation allows covering the range of $-100^{\circ}<\alpha<100^{\circ}$. The experimental results on real antagonistic devices, however, reveal lower folding angles due to additional forces in the devices depending on the details of fabrication. Figure 11 shows an example of bi-directional self-folding microactuation by infrared microcopy, showing reversible folding between $-44^{\circ}$ and $+40^{\circ}$. The folding motion during heating and cooling of the protagonist and antagonist can be found in videos S5 and S6 of the Supplementary Materials, respectively.

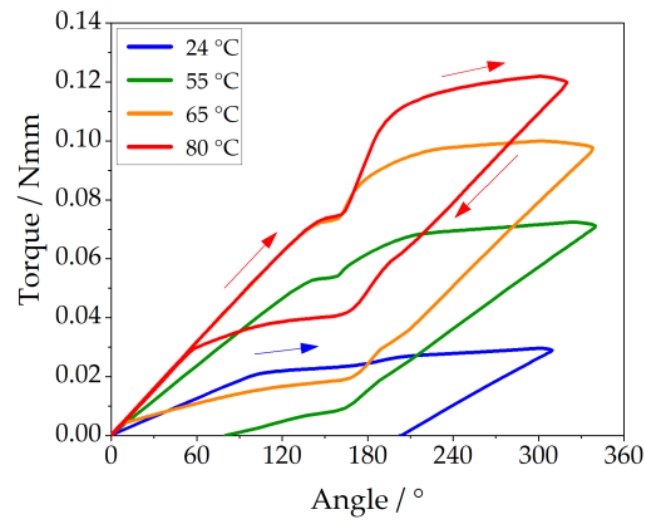

(a)

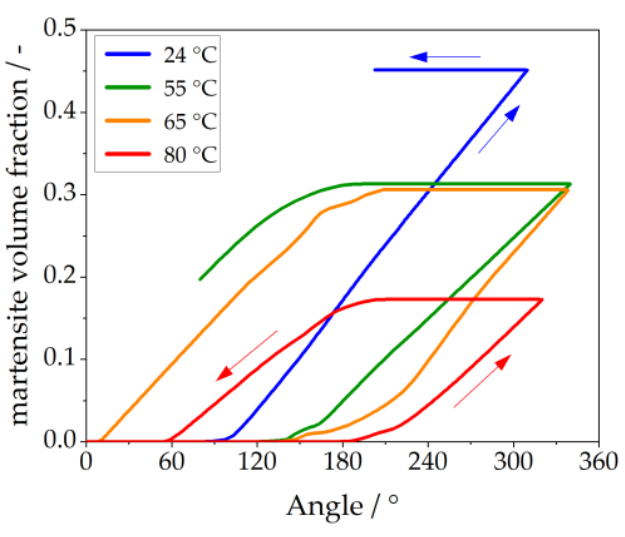

(b)

Figure 10. (a) FEM simulation of torque versus folding angle of a single microactuator for different homogeneous temperatures as indicated; (b) corresponding local martensite volume fraction versus folding angle in the bending region.
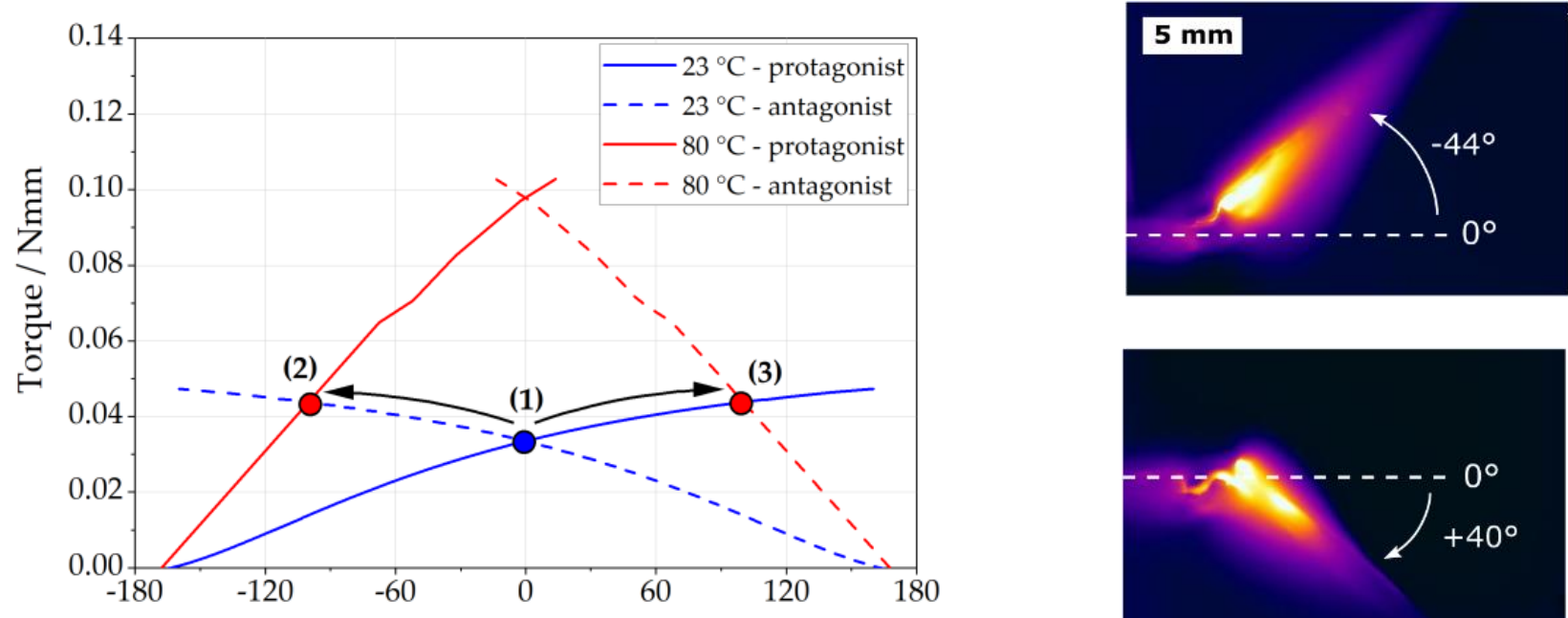

Folding Angle / ${ }^{\circ}$

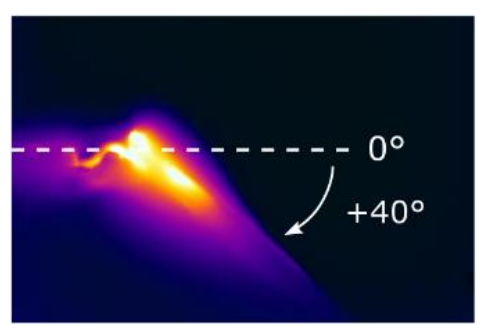

Figure 11. Torque versus folding angle characteristics of the protagonist and antagonist of an antagonistic microactuator for the case of pre-deflection by $360^{\circ}$. By heating either the protagonist or antagonist, the equilibrium point moves from (1) to either (2) or (3). 


\section{Conclusions}

We present the design, fabrication, and characterization of uni- and bi-directional origami-inspired self-folding microactuators based on micromachined SMA foils. The basic design consists of two triangular tiles that are interconnected by double-beam-shaped SMA microactuators, forming active hinges that are controlled by Joule heating. A macromodel describing the engineering stress-strain characteristics of the SMA foil is set up and implemented in an FEM tool to simulate the torque and bending angles of corresponding SMA microactuators in the temperature range of actuation. For fabrication, we follow a rapid prototyping approach using laser cutting, separate heat treatment, and hybrid integration. Upon further miniaturization, these process steps will be replaced by a monolithic fabrication scheme. Characterization concentrates on uni-directional as well as bi-directional self-folding. Experiments on folding angles versus heating power on single microactuators demonstrate the self-folding and tunability of the angular range between $30^{\circ}$ up to $180^{\circ}$, depending on the gravitational force of the tiles. The finite element simulations qualitatively describe the main features of the observed torque-folding angle characteristics including the temperature dependence of stiffness, critical stress for intermartensitic transformation, and stress-induced formation of martensite. Based on this information, the performance of antagonistic microactuators including maximum torque and bi-directional folding range is predicted. First demonstrator devices reveal bi-directional actuation in the range of $-44^{\circ}$ and $+40^{\circ}$. This range remains well below the predicted limit of $\pm 100^{\circ}$, which indicates that there is considerable room for design improvements. Possible design extensions could include cascaded designs of folding microactuators and, e.g., additional magnetic forces to support self-folding. Further downscaling and increasing the number of tiles will enable a variety of functional shapes and motions based on programmable folding patterns. Possible applications are foreseen, e.g., in programmable adaptive microoptics and microfluidics.

Supplementary Materials: The following videos S1-S6 are available online at https: / www.mdpi. com/article/10.3390/act10080181/s1. Video S1: Animation of unfolding and martensite phase fraction at $24{ }^{\circ} \mathrm{C}$ (24d-martensitefraction.mp4); Video S2: Animation of unfolding and von Mises stress in the bending region at $24{ }^{\circ} \mathrm{C}(24 \mathrm{~d}$-vonMises.mp4); Video S3: Animation of unfolding and martensite phase fraction at $80{ }^{\circ} \mathrm{C}$ (80g-martensitefraction.mp4); Video S4: Animation of unfolding and von Mises stress in the bending region at $80^{\circ} \mathrm{C}$ (80g-vonMises.mp4); Video S5: Video of the folding motion during heating of the protagonist (protagonist-heating+cooling.mp4); Video S6: Video of the folding motion during heating of the antagonist (antagonist-heating+cooling.mp4).

Author Contributions: Conceptualization, M.K. and F.W., methodology, data curation, formal analysis, and visualization, L.S., simulations, G.K.T., writing — original draft preparation, L.S. and G.K.T., writing - review and editing, M.K. and F.W. All authors have read and agreed to the published version of the manuscript.

Funding: This research was funded by the German Research Foundation (Deutsche Forschungsgemeinschaft, DFG) within the priority program "Cooperative Multistage Multistable Microactuator Systems" (SPP2206).

Institutional Review Board Statement: Not applicable.

Data Availability Statement: The data presented in this study are available on request from the corresponding author. The data are not publicly available, because they are used in ongoing research.

Acknowledgments: We thank S. Wadhwa for performing the laser structuring of NiTi foils and $\mathrm{H}$. Fornasier for UV lithography processing.

Conflicts of Interest: The authors declare no competing interest. 


\section{Appendix A}

Table A1. List of parameters for finite element simulations.

\begin{tabular}{ccc}
\hline Parameter & Abbreviation & Value \\
\hline Martensite Start Temperature & $M_{S}$ & $19^{\circ} \mathrm{C}$ \\
Martensite Finish Temperature & $M_{f}$ & $9{ }^{\circ} \mathrm{C}$ \\
Austenite Start Temperature & $A_{s}$ & $52^{\circ} \mathrm{C}$ \\
Austenite Finish Temperature & $A_{f}$ & $62^{\circ} \mathrm{C}$ \\
R-phase Peak Temperature & $R_{p}$ & $45^{\circ} \mathrm{C}$ \\
& & $12.9 \mathrm{GPa}\left(\right.$ at $\left.24^{\circ} \mathrm{C}\right)$ \\
Young's Modulus (starting phase) & $E_{A}$ & $21.4 \mathrm{GPa}\left(\right.$ at $\left.40^{\circ} \mathrm{C}\right)$ \\
& & $24.3 \mathrm{GPa}\left(\mathrm{at} 55^{\circ} \mathrm{C}\right)$ \\
Clausius Clapeyron coefficient & $C_{M}$ & $32.5 \mathrm{GPa}\left(\mathrm{at} 80^{\circ} \mathrm{C}\right)$ \\
Maximum uniaxial transformation strain & $\varepsilon_{t r}^{\text {max }}$ & $6.6 \mathrm{MPa} / \mathrm{K}$ \\
Critical transformation start stress $(\mathrm{A} \rightarrow \mathrm{M})$ & $\sigma_{S C R}$ & $0.035\left(\mathrm{at} 24^{\circ} \mathrm{C}\right)$ \\
Poisson ratio & $v$ & $\sim 5025\left(40{ }^{\circ} \mathrm{C}, 55^{\circ} \mathrm{C}, 80^{\circ} \mathrm{C}\right)$ \\
& & 0.33
\end{tabular}

\section{References}

1. Miskin, M.Z.; Dorsey, K.J.; Bircan, B.; Han, Y.; Muller, D.A.; McEuen, P.L.; Cohen, I. Graphene-based bimorphs for micron-sized, autonomous origami machines. Proc. Natl. Acad. Sci. USA 2018, 115, 466-470. [CrossRef]

2. Tolley, M.T.; Felton, S.M.; Miyashita, S.; Aukes, D.; Rus, D.; Wood, R.J. Self-folding origami: Shape memory composites activated by uniform heating. Smart Mater. Struct. 2014, 23, 94006. [CrossRef]

3. Jiang, M.; Gravish, N. Reconfigurable laminates enable multifunctional robotic building blocks. Smart Mater. Struct. 2021, 30. [CrossRef]

4. Cromvik, C.; Eriksson, K. Airbag Folding Based on Origami Mathematics; Chalmers University of Technology: Göteborg, Sweden, 2006.

5. Baek, S.-M.; Yim, S.; Chae, S.-H.; Lee, D.-Y.; Cho, K.-J. Ladybird beetle-inspired compliant origami. Sci. Robot. 2020, 5. [CrossRef]

6. Ho, M.; Kim, Y.; Cheng, S.S.; Gullapalli, R.; Desai, J.P. Design, development, and evaluation of an MRI-guided SMA springactuated neurosurgical robot. Int. J. Rob. Res. 2015, 34, 1147-1163. [CrossRef] [PubMed]

7. Momeni, F.; M.Mehdi Hassani.N, S.; Liu, X.; Ni, J. A review of 4D printing. Mater. Des. 2017, 122, 42-79. [CrossRef]

8. Peraza-Hernandez, E.A.; Hartl, D.J.; Malak, R.J., Jr.; Lagoudas, D.C. Origami-inspired active structures: A synthesis and review. Smart Mater. Struct. 2014, 23, 94001. [CrossRef]

9. Rogers, J.; Huang, Y.; Schmidt, O.G.; Gracias, D.H. Origami MEMS and NEMS. MRS Bull. 2016, 41, 123-129. [CrossRef]

10. Peraza-Hernandez, E.A.; Hartl, D.J.; Malak, R.J., Jr. Design and numerical analysis of an SMA mesh-based self-folding sheet. Smart Mater. Struct. 2013, 22, 94008. [CrossRef]

11. Shin, B.H.; Jang, T.; Ryu, B.-J.; Kim, Y. A modular torsional actuator using shape memory alloy wires. J. Intell. Mater. Syst. Struct. 2016, 27, 1658-1665. [CrossRef]

12. Ho, M.; McMillan, A.; Simard, J.M.; Gullapalli, R.; Desai, J.P. Towards a Meso-Scale SMA-Actuated MRI-Compatible Neurosurgical Robot. IEEE Trans. Robot. 2011, 2011, 1-10. [CrossRef] [PubMed]

13. Boyvat, M.; Koh, J.-S.; Wood, R.J. Addressable wireless actuation for multijoint folding robots and devices. Sci. Robot. 2017, 2 [CrossRef]

14. Torres-Jara, E.; Gilpin, K.; Karges, J.; Wood, R.J.; Rus, D. Composable flexible small actuators built from thin shape memory alloy sheets. IEEE Robot. Automat. Mag. 2010, 17, 78-87. [CrossRef]

15. Hawkes, E.; An, B.; Benbernou, N.M.; Tanaka, H.; Kim, S.; Demaine, E.D.; Rus, D.; Wood, R.J. Programmable matter by folding. Proc. Natl. Acad. Sci. USA 2010, 107, 12441-12445. [CrossRef]

16. Paik, J.K.; Wood, R.J. A bidirectional shape memory alloy folding actuator. Smart Mater. Struct. 2012, 21, 65013. [CrossRef]

17. Jeong, J.W.; Yoo, Y.I.; Shin, D.K.; Lim, J.H.; Kim, K.W.; Lee, J.J. A novel tape spring hinge mechanism for quasi-static deployment of a satellite deployable using shape memory alloy. Rev. Sci. Instrum. 2014, 85, 25001. [CrossRef] [PubMed]

18. Otsuka, K. (Ed.) Shape Memory Materials; Cambridge Univ. Press: Cambridge, UK, 1999; ISBN 052144487X.

19. Miyazaki, S.; Otsuka, K. Deformation and transition behavior associated with the R-phase in Ti-Ni alloys. MTA 1986, 17, 53-63. [CrossRef]

20. Šittner, P.; Landa, M.; Lukáš, P.; Novák, V. R-phase transformation phenomena in thermomechanically loaded NiTi polycrystals. Mech. Mater. 2006, 38, 475-492. [CrossRef]

21. Jaber, M.B.; Smaoui, H.; Terriault, P. Finite element analysis of a shape memory alloy three-dimensional beam based on a finite strain description. Smart Mater. Struct. 2008, 17, 45005. [CrossRef] 
22. Jaber, M.B. User Manual of the UMAT Subroutine for an SMA Strain Based Constitutive Model. Available online: www. researchgate.net/publication/280729999_User_Manual_of_the_UMAT_Subroutine_for_an_SMA_strain_based_constitutive_ model (accessed on 27 July 2021).

23. Jaber, M.B.; Mehrez, S.; Ghazouani, O. A 1D constitutive model for shape memory alloy using strain and temperature as control variables and including martensite reorientation and asymmetric behaviors. Smart Mater. Struct. 2014, 23, 95026. [CrossRef]

24. ABAQUS. Abaqus Theory Manual V6.11: Mechanical Constitutive Models. Dassault Systèmes Simulia Corp. 2006. Available online: 130.149.89.49:2080/v6.11/books/hhp/default.htm (accessed on 27 July 2021).

25. Bathe, K.-J. Finite Element Procedures; Prentice Hall: Englewood Cliffs, NJ, USA, 1996; ISBN 978-0133014587.

26. Gall, K.; Sehitoglu, H. The role of texture in tension-compression asymmetry in polycrystalline NiTi. Int. J. Plast. 1999, 15, 69-92. [CrossRef]

27. Seigner, L.; Bezsmertna, O.; Fähler, S.; Tshikwand, G.; Wendler, F.; Kohl, M. Origami-Inspired Shape Memory Folding Microactuator. In Proceedings of the 1st International Electronic Conference on Actuator Technology: Materials, Devices and Applications, Sciforum.net, 23-27 November 2020; MDPI: Basel, Switzerland, 2020; p. 8480. 